\title{
MEASUREMENT OF CABBAGE (BRASSICA OLERACEA L.) ROOT TREATED WITH BENEFICIAL MICROBIAL CONSORTIA THROUGH COMPUTER VISION TECHNOLOGY
}

\author{
CoBos-TorRes, J. C. $.^{*}-$ AlvarEZ-VERA, M. ${ }^{2}-$ FloRES-VASQUEZ, C. ${ }^{3}$ \\ ${ }^{1}$ Postgraduate Leadership, Electrical Engineering Career, Research Group in Visible Radiation \\ and Prototyping GIRVyP and Research Group in Embedded Systems and Artificial Vision in \\ Architectural, Agricultural, Environmental and Automatic Sciences (SEVA4CA), Catholic \\ University of Cuenca, Cuenca, Ecuador \\ ${ }^{2}$ Postgraduate Leadership, Environmental Engineering Career, Research Group in Embedded \\ Systems and Artificial Vision in Architectural, Agricultural, Environmental and Automatic \\ Sciences (SEVA4CA), Catholic University of Cuenca, Cuenca, Ecuador \\ ${ }^{3}$ Electrical Engineering Career, Research Group in Visible Radiation and Prototyping \\ (GIRVyP), Catholic University of Cuenca, Cuenca, Ecuador \\ *Corresponding author \\ e-mail: juan.cobos@ucacue.edu.ec; phone: +593-96-263-5040
}

(Received $8^{\text {th }}$ Jun 2021; accepted $4^{\text {th }}$ Sep 2021)

\begin{abstract}
This study aimed to measure the root length and surface area of cabbage plants (Brassica oleracea L.) by using computer vision technology (RGB Camera). This investigation was conducted by a transdisciplinary team made up of researchers from the agronomy, environmental, electronic, and computer vision fields. Researchers from the first two fields were responsible for obtaining biofertilizers by applying beneficial microbial consortia to household organic waste; in addition, to planting cabbage seeds with different amounts of biofertilizer. The last two fields were responsible for developing a system that would measure root length and root surface area by using image analysis. As a result, plants enriched with $20 \%$ and $25 \%$ of biofertilizers showed better performance in root length. In comparison, these plants, compared to the control group had $63 \%$ more surface area root and were $61 \%$ longer root. These results show that biofertilizers should be applied in adequate amounts to obtain higher root length and surface area. This is important since plant roots absorb water and nutrients for plant development and these functions depend on the length of roots and fine roots. In addition, it should be emphasized that conventional root measurement techniques are time-consuming and provide inaccurate data. This research presents a methodology where computer vision technology is applied to optimize the measurement process, providing accurate data in less time.
\end{abstract}

Keywords: biofertilizers, root length, root surface area, beneficial soil microorganisms, camera

\section{Introduction}

Due to global population growth, efforts to increase food production are imperative in order to meet constant food demand. Though the roots of plants - which absorb water and soil nutrients needed for plant development and crop performance - play a fundamental role in agricultural production, they are sometimes undervalued. Although even today there are different criteria about roots measurement, Novoplansky (2019) affirms that until recently the study of roots has been neglected, despite its importance for plant life.

Roots secrete metabolites into the rhizosphere (the soil zone around the roots, which is also known to reserve microorganisms (Gouda et al., 2018) that fulfill primary functions in plant nutrition) intervening in several processes (Vives-Peris et al., 2019). 
Chemical fertilizers are used to improve plant development and performance; however, their permanent use represents an important economic outlay, and their indiscriminate use affects the soil and the environment.

To replace chemical fertilizers, one of the greatest gifts that nature offers are biofertilizers; they are composed of microorganisms that benefit the supply of nutritional elements to plants, therefore, contributing to improve their development and regulate their physiology (Kumar et al., 2018). Microbial biofertilizers are considered an alternative to chemical fertilizers, due to their high potential to enhance agricultural production (Mahanty et al., 2017) and preserve crop productivity, by efficiently using fertilizers without affecting the environment, which is key to agricultural systems (Paungfoo-Lonhienne et al., 2019) and sustainable development

The application of bioinoculants, with fertilizing properties, to soil has attracted great interest in recent times due to the environmental and economic advantages they provide and also due to their impact on the rhizosphere. Biofertilizers arise as potentially ecofriendly products that benefit agricultural crop production. They are applied in seeds or to the soil, improving nutrient availability, thus enhancing the growth and yield of cultivated plants (Bisht et al., 2016).

Automation and computer vision technology are viable options to be used in agriculture for studying the phenology of plants and crop yield precisely, as well as for time and resource optimization. Root size measurements have varied over the last decades, each quantification method has evolved in measurement reliabilities and the variation between results (Judd et al., 2015). Basic methods as drawing roots (Weaver et al., 1922), window (Böhm, 1979) trench (Schuurman and Goedewaagen, 1965), and currently in the last four decades using cameras (Ottman and Timm, 1984; Villordon et al., 2012; Wang et al., 2019) have made it possible to facilitate and improve the quantification of roots. Quantification using cameras, despite reducing the time required for measurement and avoiding human subjectivism, still takes considerable time when it comes to quantifying studies with repetitions. It is necessary to automate the process so that several measurements can be executed serially, reducing the measurements time. In addition, it is important to reduce costs by using Opensource software and low-cost hardware.

Cabbage (Brassica oleracea L.), an important element in people's diet, is part of a wide variety of vegetable plants that are cultivated locally. However, there is not enough research that focuses on the study of cabbage roots. Traditional methods applied have provided inaccurate data due to manual registration systems. The accuracy in measuring the characteristics of the root system is important for plant phenotyping (Van Dusschoten et al., 2016). Roots are responsible for water and nutrient absorption: processes required for photosynthesis and agricultural production (Tracy et al., 2020).

By means of technology, agriculture can be enhanced through automation for small field farming, allowing to achieve high precision, efficiency, and low costs (Tian et al., 2020). In agriculture, artificial intelligence becomes a key technique for solving different problems (Bannerjee et al., 2018); it is considered to be a feasible solution to increase food production for a constantly growing population (Zha, 2020). It is possible to improve the growth and performance of plants by mixing biofertilizers. Therefore, it is important for farmers to know the synergistic effects of these bioinoculants (Agarwal et al., 2018), relying on modern measurement and quantification techniques.

This research aimed to determine the length of roots of cabbage plants, by means of digital cameras, in order to know the effect of biofertilizers on root growth. 


\section{Materials and methods}

This research was carried out in four stages: (i) producing biofertilizers with household organic waste by applying beneficial microbial consortia, (ii) planting cabbage seeds with different amounts of fertilizer; iii) developing a system for measuring root length and surface area by means of images and iv) measuring root of cabbage plants.

In the first stage, beneficial microorganisms were obtained from the phyllosphere of the lemon verbena plant (Aloysia citrodora). All samples were obtained from the aerial region of the plant. Beneficial microbial consortia were obtained from the parts of the plants collected, through a fermentation process. At the same time, household organic food waste was collected, that is, vegetable waste. Organic waste was composted by applying beneficial microorganisms. Likewise, vermicompost of organic waste was carried out by applying beneficial microorganisms and using Red Californian Earthworms. Polystyrene boxes of $0.40 \mathrm{~m} \times 0.30 \mathrm{~m} \times 0.32 \mathrm{~m}$ were used in both processes. In the particular process of vermicomposting, $1 \mathrm{~kg}$ of earthworms was added to organic waste and vermicompost was obtained after 90 days. In addition, beneficial microbial consortia were applied, once a week, during the process. In contrast, composting was carried out under aerobic conditions, controlling humidity and temperature, for a period of 90 days as well. Beneficial microbial consortia were applied once every 15 days to moisten the substrate. The resulting material from both processes was black and smelled like petrichor. Finally, $5 \mathrm{~kg}$ of compost and $5 \mathrm{~kg}$ of vermicompost were mixed in a thoroughly cleaned container, in equal proportions, obtaining the desired biofertilizer. Figure 1 shows the entire process.

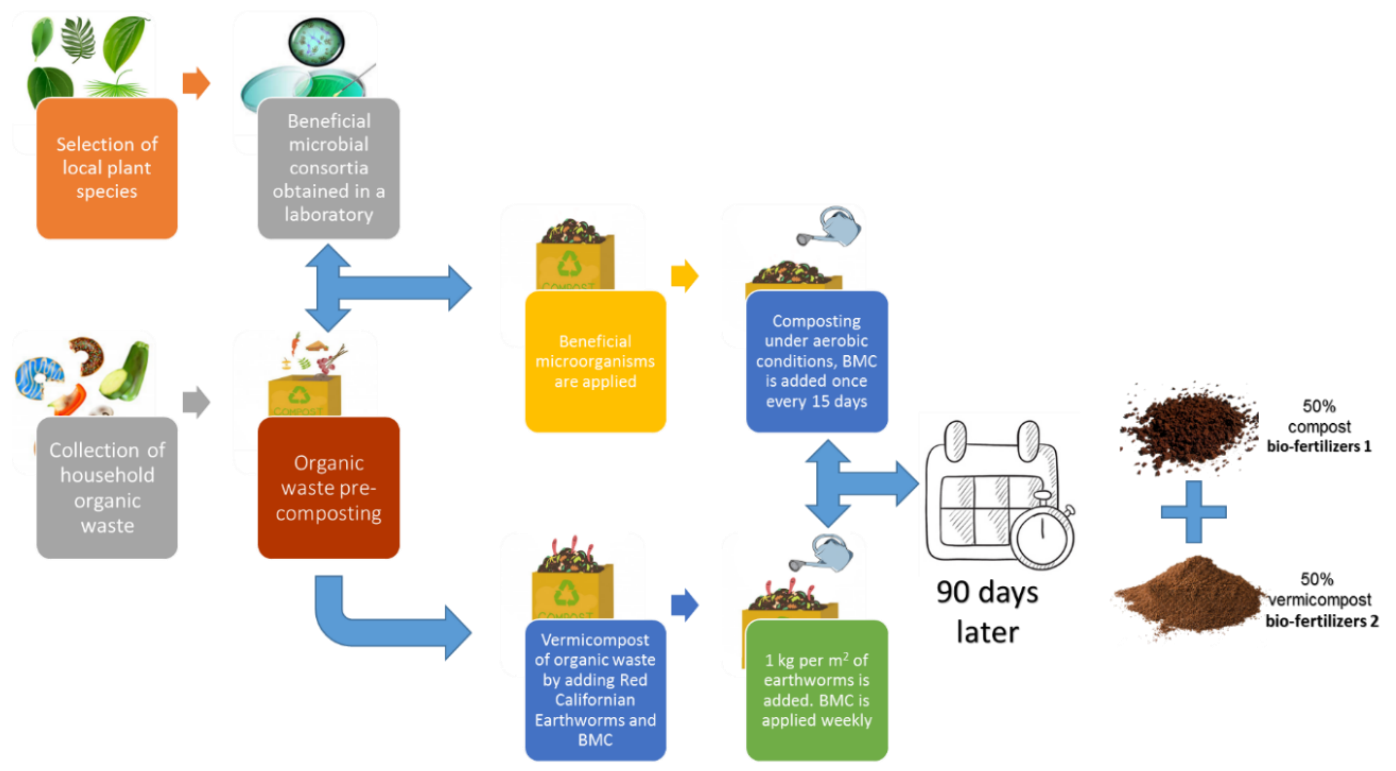

Figure 1. Process for biofertilizer production

In the second stage, cabbage seeds (this plant type was selected because it is a traditional crop in our environment) were planted in seedbeds made with cups. In each cup, $100 \mathrm{~g}$ of commercial peat (material used for rooting) and different concentrations of biofertilizer were added. These cups were holes in the bottom to ensure adequate drainage. Biofertilizer was not applied to one treatment for control purposes. A 
randomized complete block design was applied. Seven treatments and four repetitions were carried out. Table 1 shows the different treatments and the percentage of biofertilizer added.

Table 1. Treatment details

\begin{tabular}{c|c}
\hline Treatment & Biofertilizer percentage \% \\
\hline T1 & 0 \\
T2 & 5 \\
T3 & 10 \\
T4 & 15 \\
T5 & 20 \\
T6 & 25 \\
T7 & 30 \\
\hline
\end{tabular}

The seeds were sown in the city of Gualaceo, Ecuador (2॰53'10.4”S $\left.78^{\circ} 46^{\prime} 45.2^{\prime \prime} \mathrm{W}\right)$. Planting schematization and climatic factors can be seen in Figure 2.

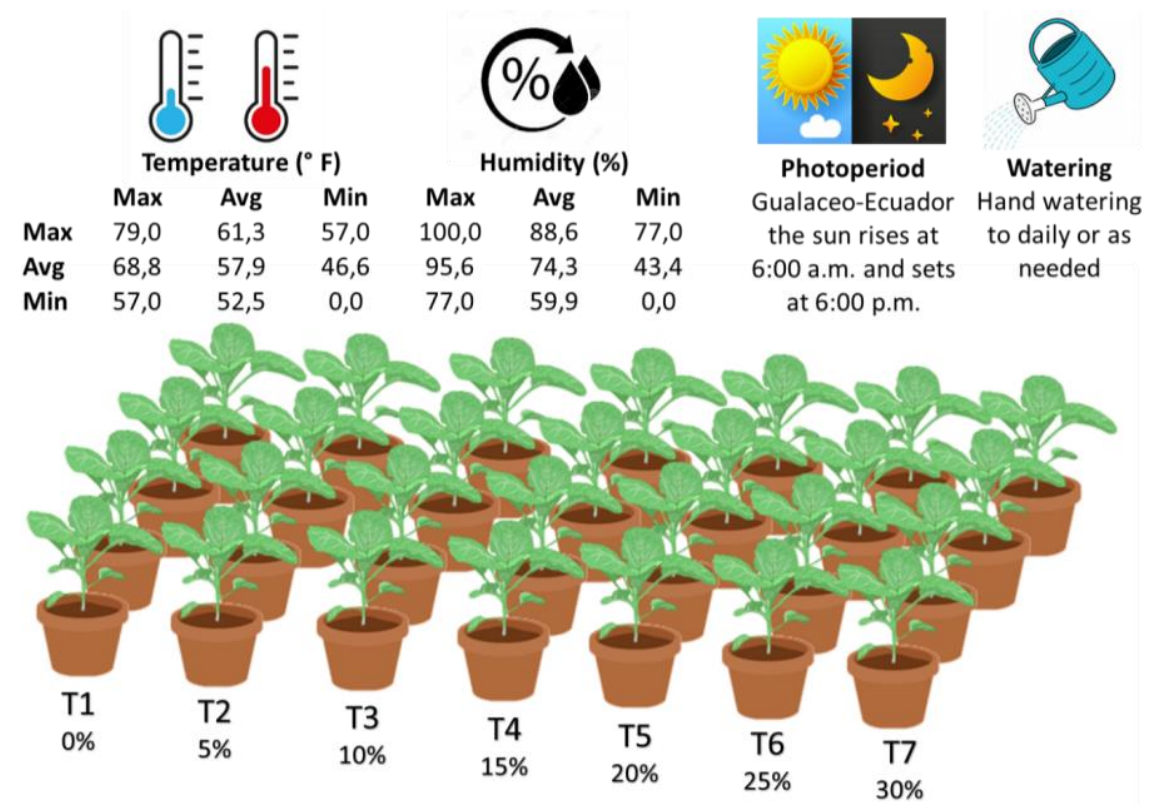

Figure 2. Treatments performed with different amounts of biofertilizer

For developing the measurement system, the third stage was divided into three substages: (a) defining a methodology for taking photographs of cabbage roots; (b) developing an aberration correction algorithm; (c) developing root length and surface area measurement algorithm using digital cameras.

a) The system was composed of a Samsung Galaxy S7 Edge cellphone with a digital camera, with a 12 MP (f1.7) resolution and optical image stabilization. The phone was placed over a desk using a cellphone holder, aiming to position the phone in such a way that the image would be framed within a black construction paper, in order to reduce perspective distortion. This system can be seen in Figure 3. 
In this stage, the roots were cleaned and exposed. The plants were grown in disposable plastic cups, which allowed for their easy extraction. The plants and soil- were soaked in a bucket filled with water for one day; any traces of soil in fine roots were washed. Once the fine roots were clean, they were placed over black construction paper for measuring.

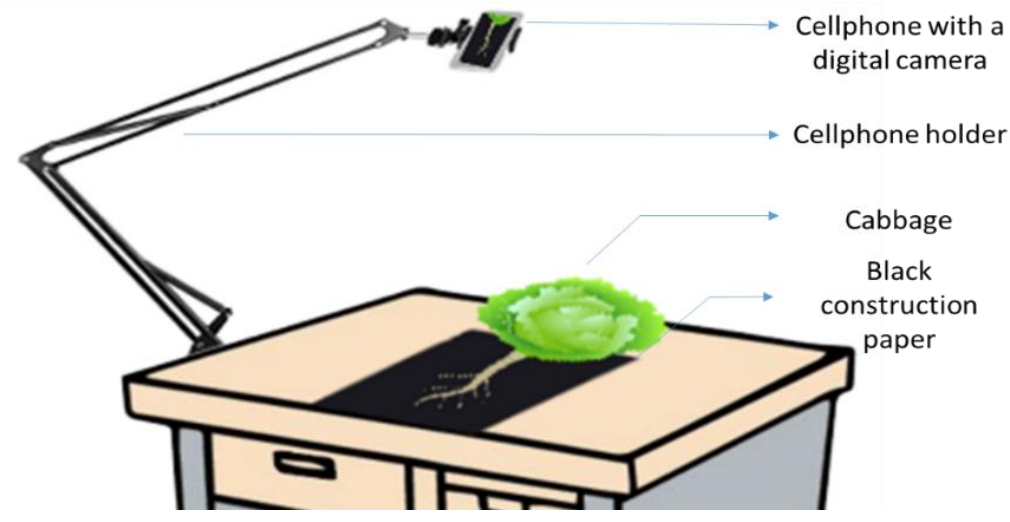

Figure 3. Methodology outline for photographing

b) The development of the algorithm for cabbage root length and surface area calculation was divided into five stages: first, obtaining the images in an RGB format by means of a camera; second, correcting perspective distortion; third, selecting pixels that correspond to the roots, by means of thresholding; fourth, counting the number of pixels of the region of interest; and fifth, calibrating the pixel $/ \mathrm{cm}$ ratio to determine the perimeter and surface area. The outline proposed can be observed in Figure 4.

\begin{tabular}{|l|}
\hline RGB image acquisition \\
• Roots are photographed with a digital camera \\
• Photographs are labeled and stored \\
\hline Image calibration \\
• Optical aberrations are corrected \\
- Perspective distortion is corrected \\
\hline Thresholding \\
\hline - Upper threshold and lower threshold are set \\
•The region of interest is segmented \\
\hline Pixel count \\
\hline -Images are binarized \\
•White pixels are counted \\
\hline Perimeter and surface area \\
\hline - Pixel/centimeter ratio is determined \\
\hline
\end{tabular}

Figure 4. Schematization of the development of an algorithm used for cabbage root size quantification 
c) For this process, a ratio that could quantify the pixels per a given metric, within the image, was defined. This is known as pixels per metric ratio. It should be noted that first, the imaging (knowledge of its intrinsic and extrinsic parameters) and distortion correction systems were pre-calibrated. After precalibration, calibration was carried out using a reference object: size A4, black, construction paper. This reference object had all the required attributes: its dimensions (width and length) were known in centimeters and it could be easily identified by its shape.

For the statistical analysis, the graphical method proposed by (Tukey, 1977) called box-and-whisker graph will be used. This shows a statistical summary of median, quartiles, range, and possibly extreme values. Additionally, the variation proposed by (Altman, 1990) (McGill et al., 1978) will be calculated including confidence intervals for the median is provided by means of notches surrounding the medians. Finally, through moving average trend lines of consecutive observations, fluctuations in the data can be smoothed to see the pattern or trend more clearly.

\section{Results}

As previously detailed in the materials and methods section, this research was developed in four stages, which were carried out to successfully achieve the proposed objective. The results of each stage are detailed in the following subsections.

\section{Biofertilizers production}

The lemon verbena plant (Aloysia citrodora), cultivated in our environment, was used to obtain beneficial microbial consortia. The concentration of microorganisms in colony-forming units per milliliter of solution $(\mathrm{CFU} / \mathrm{mL})$, is presented in Table 2.

Table 2. Composition of beneficial microbial consortia

\begin{tabular}{c|c|c}
\hline $\mathbf{N}^{\circ}$ & Microorganism & $\mathbf{C F U} / \mathbf{m L}$ \\
\hline 1 & Acinetobacter sp. & 0.34298 \\
2 & Aeromonas sp. & 129.475 \\
3 & Alcaligenes sp. & 28.472 \\
4 & Bacillus cereus & 202.941 \\
5 & Bacillus subtilis & 121.231 \\
6 & Candida sp. & 0.98246 \\
7 & Clostridium sp. & 190.928 \\
8 & Lactobacillus spp. & 0.39875 \\
9 & Listeria monocytogenes & 0.45922 \\
10 & Micrococcus sp. & 213.476 \\
11 & Pseudomonas aeruginosa & 0.6439 \\
12 & Pseudomonas fluorescens & 0.27424 \\
13 & Pseudomonas putida & 162.572 \\
14 & Saccharomyces cerevisiae & 274.925 \\
15 & Salmonella sp. & 0.29289 \\
16 & Yarrowia lipolytica & 123.827 \\
\hline
\end{tabular}


The process began by cutting off the leaves of the plant. This can be seen in Figure $5 \mathrm{a}$. The leaves were taken to the laboratory of the Postgraduate Leadership of the Catholic University of Cuenca to obtain beneficial microbial consortia by anaerobic fermentation. The final product can be seen in Figure $5 b$. At the same time, researchers collected organic waste from their homes. By a visual inspection, it was determined that most of the waste consisted of food waste. Once $20 \mathrm{~kg}$ of organic waste were collected, compost was prepared by inoculating it with previously obtained beneficial microorganisms. This can be seen in Figure 5c. Similarly, in a separate box, vermicompost was made by applying $1 \mathrm{~kg}$ of Red Californian Earthworms (Eisenia foetida) to the waste and inoculating it with beneficial microorganisms. As already detailed in the previous section, both composts were prepared in polystyrene boxes of $0.40 \mathrm{~m} \times 0.30 \mathrm{~m} \times 0.32 \mathrm{~m}$. This can be seen in Figure $5 d$ and $e$. Both processes were performed in ninety days. The composting process was checked and inoculated every 8 days with a liquid substrate enriched with beneficial microorganisms, which helped the process and kept the substrate moist. In contrast, the vermicomposting process was checked and inoculated every 7 days with the same liquid substrate, enriched with beneficial microorganisms. This can be seen in Figure $5 f$ and $g$. The resulting material was black, smelled like petrichor, and met the stability characteristics. Finally, a biofertilizer was produced by mixing compost and vermicompost, in a $5 \mathrm{~kg}$ of compost and $5 \mathrm{~kg}$ of vermicompost ratio. Figure $5 \mathrm{~h}$ shows the final result.

\section{Seed planting in different treatments}

Cabbage seeds were planted in seedbeds made with disposable plastic cups. Such containers were selected to facilitate plant removal. Each cup contained $100 \mathrm{~g}$ of commercial soil with different concentrations of biofertilizer (see Table 1). Plants in their seedbeds can be seen in Figure 6.

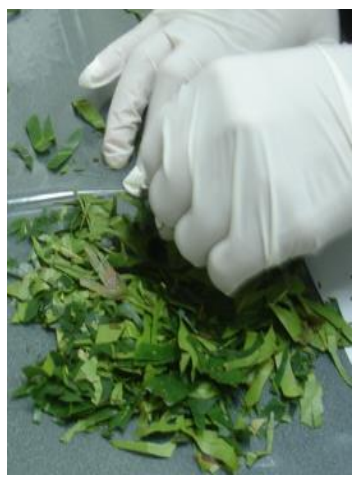

(a)

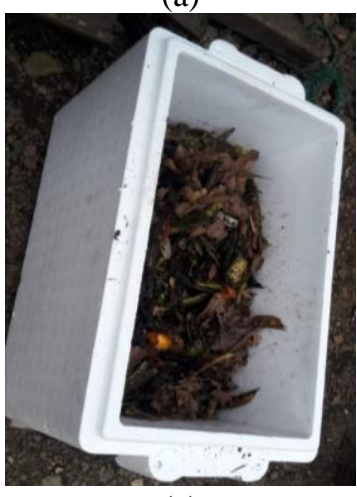

(c)

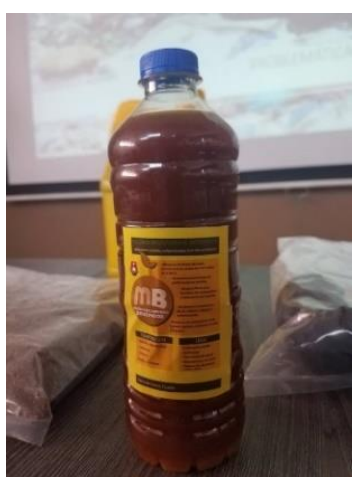

(b)

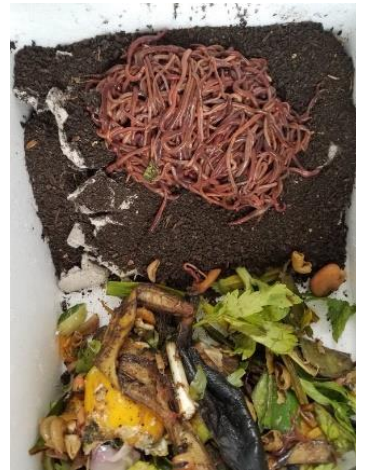

(d) 


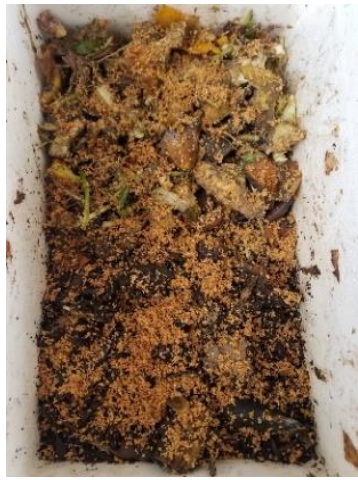

(e)

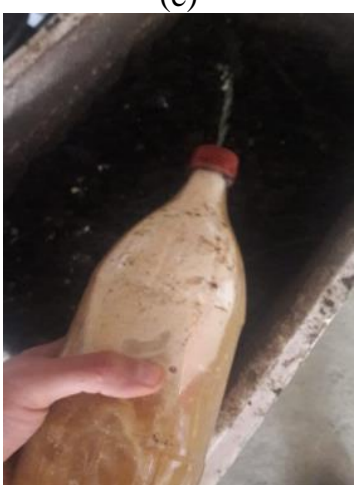

(g)

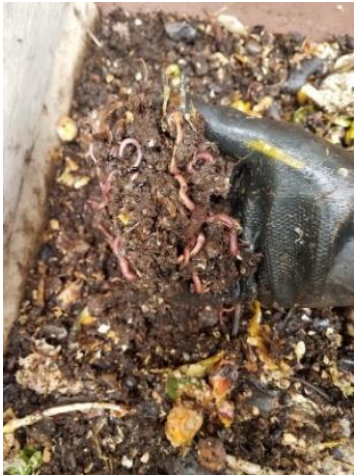

(f)

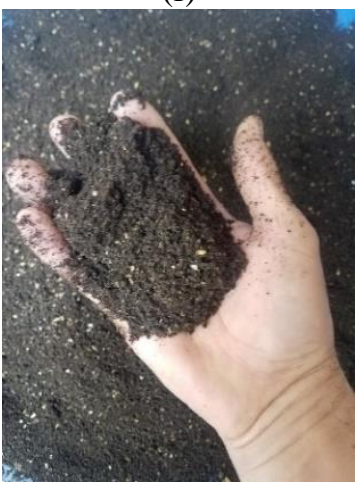

(h)

Figure 5. Stages in biofertilizer production: (a) Selection of phyllosphere microorganisms; (b) Liquid solution with beneficial microorganisms; (c) Organic waste in the composting process; (d) Vermicomposting with earthworms; (e) Compost under aerobic conditions; $(f)$ Vermicompost; $(g)$ Inoculation with beneficial microorganisms; $(h)$ Biofertilizer

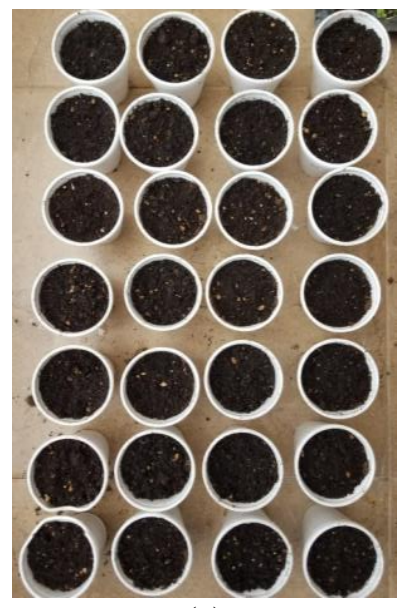

(a)

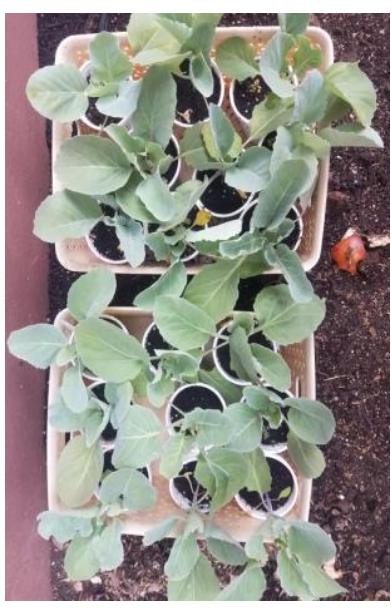

(b)

Figure 6. Cabbage seedbeds: (a) One week after planting; (b) Four weeks after planting

\section{Measurement system}

In this stage, the plants were removed from their seedbeds and soaked in a bucket filled with water, for one day. Once the soil was loose, any remaining soil was washed, cleaning the roots completely, leaving white fine roots exposed. Next, photographs were 
taken; fitting the image within the black construction paper, providing high color contrast. This was useful for the segmentation of the photographed roots performed in the following stage. This can be seen in Figure 7.

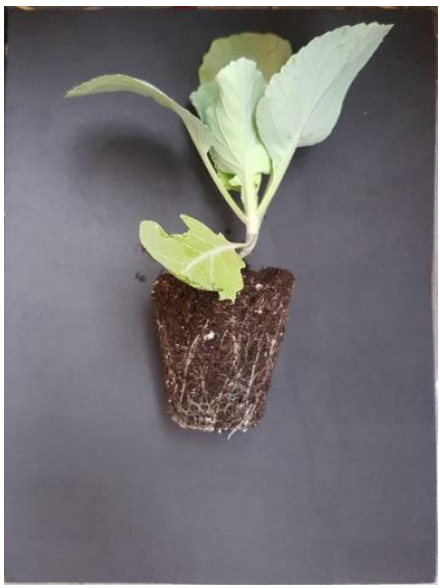

(a)

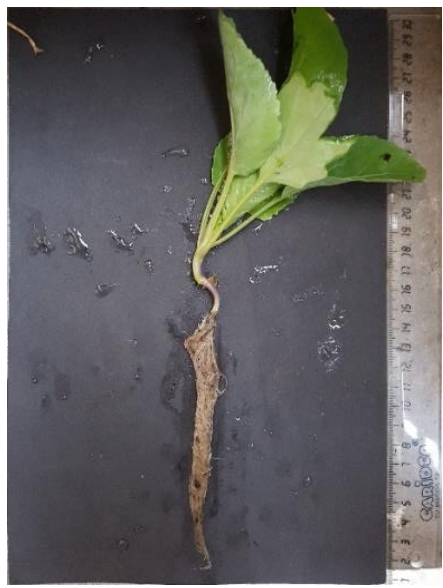

(b)

Figure 7. Preparation and cleaning of cabbage roots: (a) Plant removed from its seedbed; (b) Plant exposing its roots after being washed

\section{Root measuring}

The development of the algorithm for calculating the root length and surface area of cabbage plants was carried out by using the Python programming language and the OpenCV library. The first step was analyzing the RGB images, which were previously labeled according to the treatment. Once the analysis was completed, perspective distortion was corrected and the thresholding technique was applied to select the pixels that corresponded to the roots. The stem and leaves were removed from the image for they had white shades. This task was easily performed by means of a visual assessment that allowed to identify the parts. This can be seen in Figure 8.

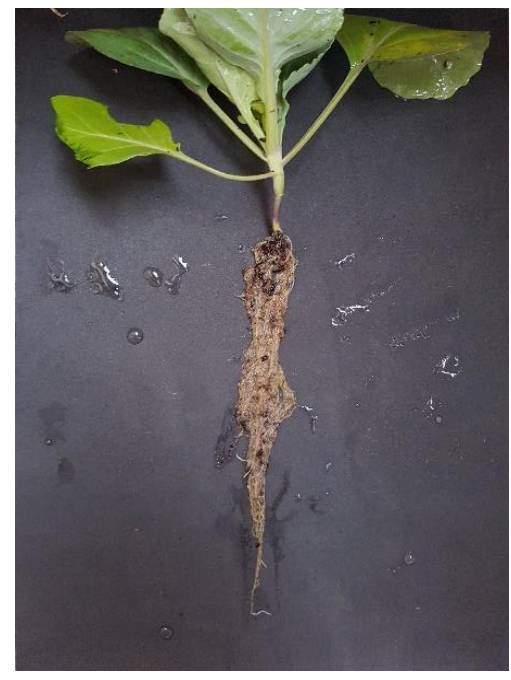

(a)

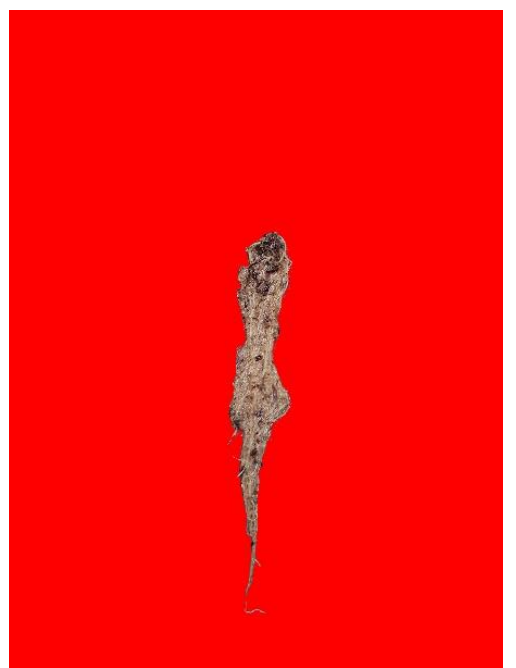

(b)

Figure 8. Image processing: (a) Perspective distortion correction; (b) Root selection by thresholding 
The pixels of the selection are quantified. The extract is the total area of the background image and the exact root surface area has to be calculated. With a scale of 136.678 pixels $/ \mathrm{cm}$ and a root pixel count of 437,878 pixels, the root surface area is $23.439 \mathrm{~cm}^{2}$.

Measurement results can be analyzed by multiple comparisons, which would allow to visualize differences between subgroups of the percentage of added biofertilizer and measurements obtained. This can be seen in Figure $9 a$ and $b$. Likewise, this information is presented in Table 3.

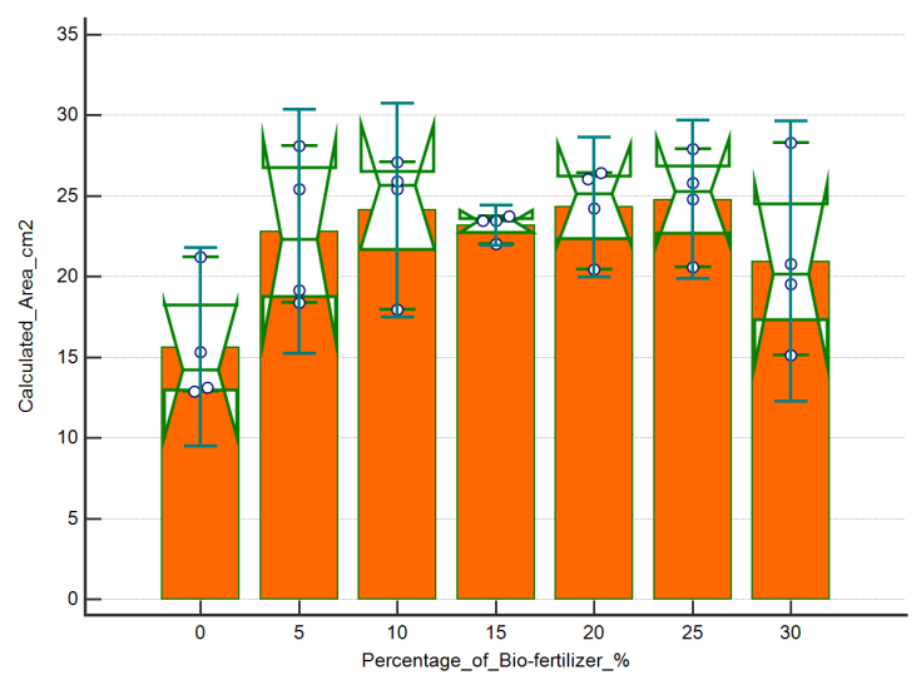

(a)

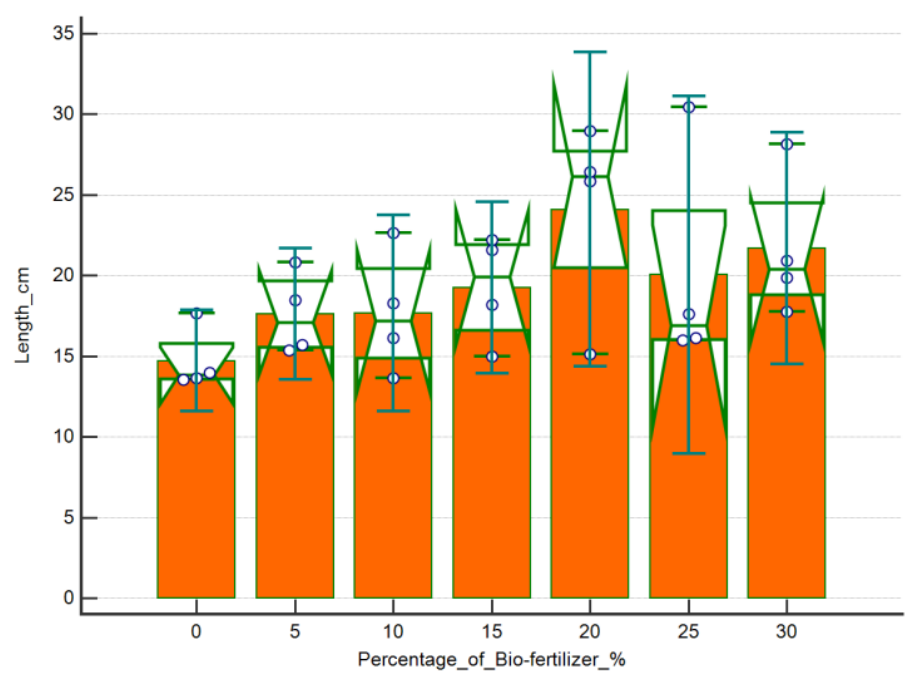

(b)

Figure 9. Root length and surface area comparison between plant samples with different contents of biofertilizer using notched box plots: (a) Surface area and (b) Perimeter

Finally, an analysis is presented in Figure 10 showing consecutive observations of both measured variables. A moving average trendline has been traced, for each variable, to smooth out the fluctuations in data, allowing for the pattern or trend to be observed more clearly. 
Table 3. Treatment measurements

\begin{tabular}{c|c|c|c|c|c}
\hline \multirow{2}{*}{ Treatment } & \multirow{2}{*}{$\mathbf{n}$} & \multicolumn{2}{|c|}{ Surface area $\mathbf{c m}^{2}$} & \multicolumn{2}{c}{ Length cm } \\
\cline { 3 - 5 } & & Mean & Median & Mean & Median \\
\hline (T1) $0 \%$ & 4 & 15.62 & 14.22 & 14.71 & 13.82 \\
(T2) 5\% & 4 & 22.78 & 22.31 & 17.60 & 17.11 \\
(T3) $10 \%$ & 4 & 24.11 & 25.68 & 17.67 & 17.21 \\
(T4) $15 \%$ & 4 & 23.18 & 23.46 & 19.25 & 19.91 \\
(T5) $20 \%$ & 4 & 24.30 & 25.16 & 24.10 & 26.15 \\
(T6) $25 \%$ & 4 & 24.78 & 25.29 & 20.04 & 16.88 \\
(T7) 30\% & 4 & 20.94 & 20.16 & 21.67 & 20.39 \\
\hline
\end{tabular}

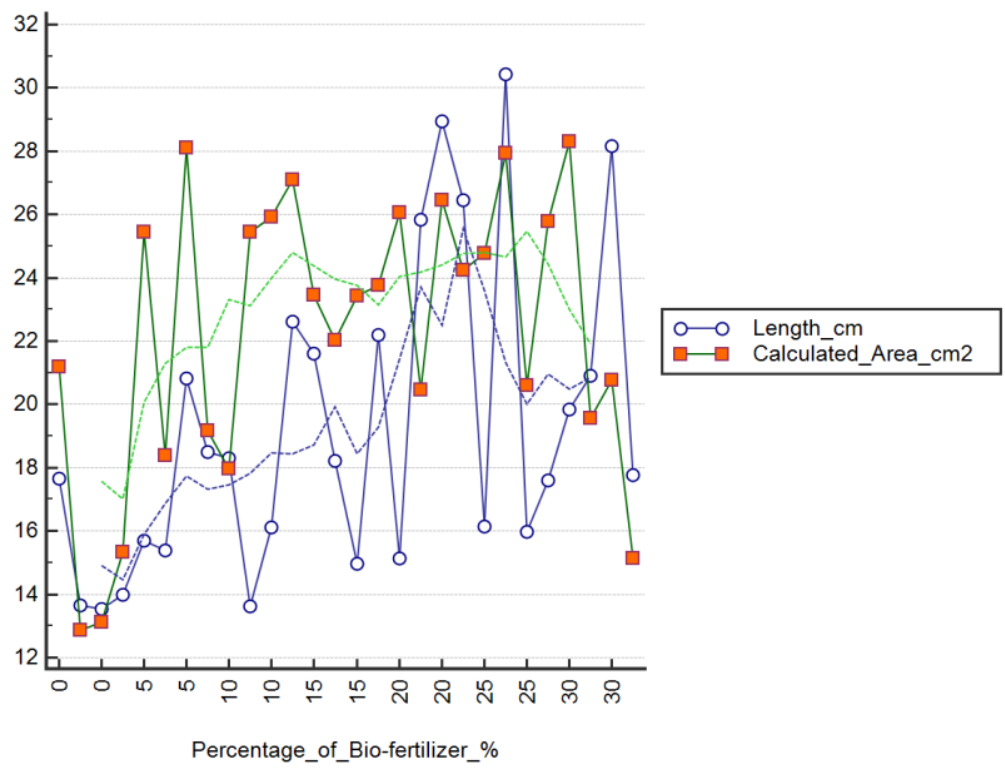

Figure 10. Root length and surface area comparison between plant samples with different contents of biofertilizer using a multiple trendline graph

\section{Measurements comparison}

In order to verify the measurements using the proposed method, these measurements were compared with the measurements made using the imageJ program (image processing program designed for scientific images). Image processing time was longer with this software but it allowed us to observe the accuracy of our measurements. For this, the Bland-Altman method quantifies the mean difference between both methods, see Figure 11.

The differences between estimates both methods were plotted against the averages of both systems for length and area, Figure 11a and $b$, respectively. Means are represented by dotted lines; $95 \%$ limits of agreement $( \pm 1.96 \mathrm{SD})$ are represented by dashed lines on the plots. Specifically, the mean biases were $0.001 \mathrm{~cm}$ with $95 \%$ limits of agreement 0.015 to $0.0017 \mathrm{~cm}$ for the length and $0.001 \mathrm{~cm}$ with $95 \%$ limits of agreement -0.013 to $0.015 \mathrm{~cm}$ for the area. The standard deviation of the residuals is $0.008 \mathrm{~cm}$ for length and $0.007 \mathrm{~cm}$ for area. 


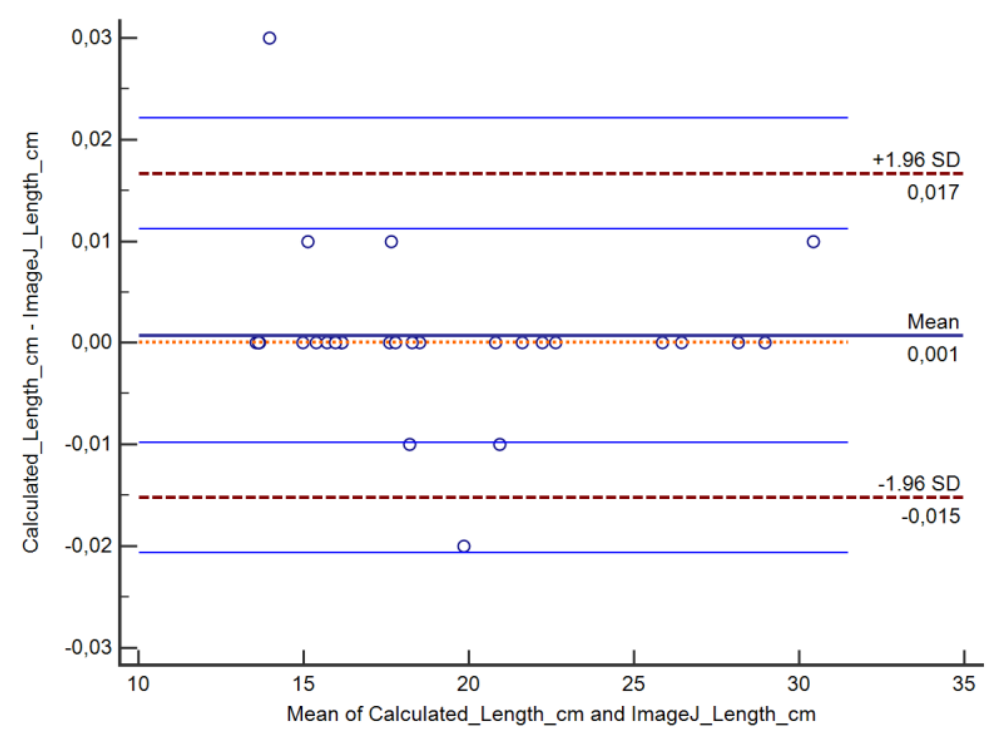

(a)

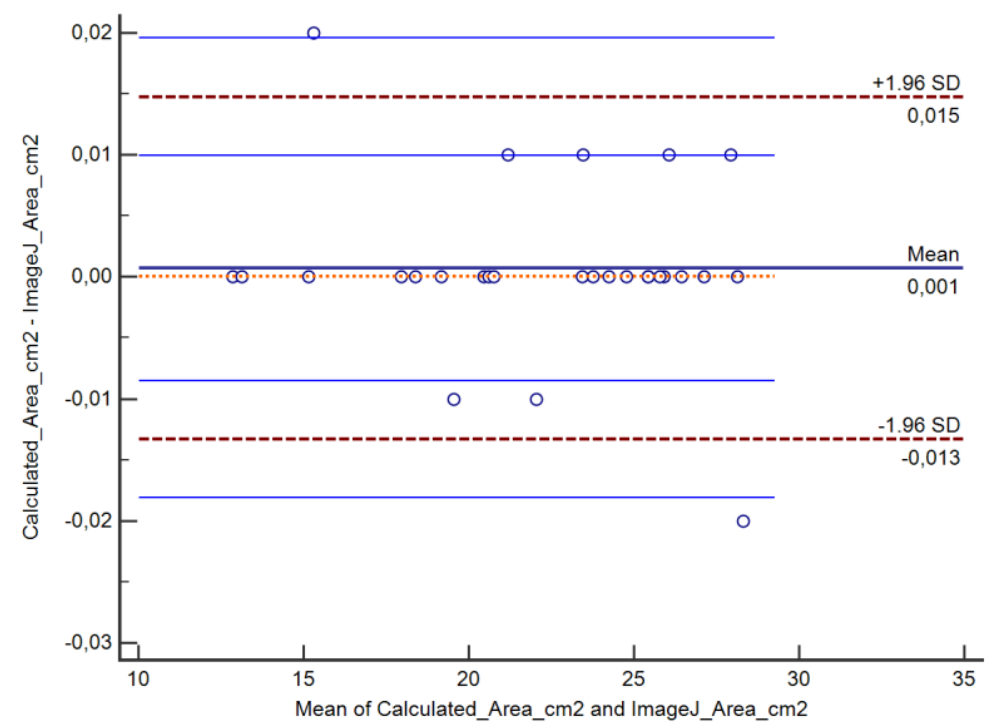

(b)

Figure 11. Bland-Altman plot showing the level of agreement between proposed method and imageJ Software: (a) length measurement and, (b) area measurement

\section{Discussion}

The application of biofertilizers benefits the root growth of crops. Several studies have previously determined benefits in the morphological characteristics of plants by using conventional measuring methods such as counting the number of leaves, measuring stem length and diameter, and measuring root length manually; thus, requiring a considerable amount of work and time, and yielding inaccurate results. This study seeks to provide a different approach: establishing a framework for measuring root length and surface area by means of technology. Applying and improving the proposal for the semi-automatic quantification of root growth of seedlings growing directly in agar plates (Betegón-Putze et al., 2018) as a reference for performing measurements outside a laboratory. This research aimed to quantify the root growth of 
seedlings, yielding accurate and rapid results on root development of plants that have been rooted in substrates with different contents of biofertilizer. Thus, the perimeter, surface area, and length of fine roots were obtained by means of image analysis.

Today, biofertilizers are used by farmers to enhance nitrogen fixation capacity. Several studies highlight that vermicompost can improve soil fertility physically, chemically, and biologically (Lim et al., 2014; Chatterjee and Debnath, 2020). It is also stated that vermicompost benefits crop growth and yield (Chatterjee R., Debnath A., 2020), increasing soil quality and plant performance (Zuo et al., 2018). Based on all these scientific statements, this research demonstrates, in a precise manner and by using software as a measuring tool, that biofertilizer application improves root length and root surface area of plants.

It is important to note that all plants inoculated with biofertilizers showed higher root growth compared with the control group. Thus, showing a larger root surface area: $45.8 \%$ (T2 vs $\mathrm{T} 1$ ), $54.4 \%$ (T3 vs $\mathrm{T} 1$ ), $48.4 \%$ (T4 vs $\mathrm{T} 1$ ), $55.6 \%$ (T5 vs $\mathrm{T} 1$ ), $58.6 \%$ (T6 vs T1) and 34.1\% (T7 vs T1). Similarly, plants with biofertilizers showed longer roots: $19.6 \%$ (T2 vs T1), $20.1 \%$ (T3 vs T1), 30.9\% (T4 vs T1), 63.8\% (T5 vs T1), 36.2\% (T6 vs T1) and 47.3\% (T7 vs T1). This can be seen in Figure 9a and $b$, where highly significant differences between treatments are evident. The application of compost and vermicompost substantially improves soil health, microorganism diversity, nutrient availability (Goswami et al., 2017), root growth, and organ development in plants. In addition, the inoculation with microorganisms fulfills fundamental functions in the rhizosphere. One study showed that bagasse compost, prepared with microorganisms, significantly improved the morphological characteristics of sorghum plants (Gopalakrishnan et al., 2019). These results are consistent with (Nascente et al., 2017), who claims that microorganisms enhance yield, promoting plant development by increasing phytomass, photosynthesis, and nutrient absorption. It is also important to highlight several particularities found in this study. First, the amount of biofertilizer seems to have a different influence on root length. Although plant containers were $10 \mathrm{~cm}$ high, the roots of plants inoculated with $20 \%$ of biofertilizer reached an average length of $24.10 \mathrm{~cm}$ and a median of $26.15 \mathrm{~cm}$; followed by plants inoculated with $30 \%, 25 \%$, and $15 \%$ of biofertilizer, where the roots reached an average length of $21.67 \mathrm{~cm}$ and a median of $20.39 \mathrm{~cm}$ in the first case; an average length of $20.04 \mathrm{~cm}$ and a median of $16.88 \mathrm{~cm}$ in the second case; and an average length of $19.25 \mathrm{~cm}$ and a median of $19.91 \mathrm{~cm}$ in the third case. While it might seem confusing, it is important to analyze Figure $9 b$ where it can be observed that plants inoculated with $15 \%$ of biofertilizer have a higher grouping in the upper quartiles, thus the notches define an upper trend. This would affirm that, in order to obtain better plant yield, the concentration of vermicompost must be applied in moderation (Lim et al., 2014). The second particularity observed is how the amount of biofertilizer influences root surface area. Plants inoculated with $25 \%$ of biofertilizer reached an average surface area of $24.78 \mathrm{~cm}^{2}$ and a median of $25.29 \mathrm{~cm}^{2}$; followed by plants inoculated with $20 \%, 10 \%$, and $15 \%$ of biofertilizer, where the roots reached an average surface area of $24.30 \mathrm{~cm}^{2}$ and a median of $25.16 \mathrm{~cm}^{2}$ in the first case; an average area of $24.11 \mathrm{~cm}^{2}$ and a median of $25.68 \mathrm{~cm}^{2}$ in the second case; and an average area of $23.18 \mathrm{~cm}^{2}$ and a median of $23.46 \mathrm{~cm}^{2}$ in the third case. Vermicompost improves soil water retention as well as plant growth regulator production (Gupta et al., 2019), which influence root development. It can be seen in Figure 9a that the notches define a higher trend for all these cases. 
It could be stated that biofertilizer content between $15 \%$ and $25 \%$ is important. This can be observed in Figure 10, where trend lines of both length and surface area show an upward tendency in the range defined above. While this does not fully agree with Ahmadpour and Armand (2020), the results are similar. They recommend the application of compost and vermicompost in a ratio of $30 \%$ of the total soil weight to improve the physiological and morphological characteristics of tomato plants under greenhouse conditions. First, it is a different crop and the methodology they used may differ in results, since in the cleaning stage there was an involuntary loss or separation of root segments. In addition, these involuntary losses could also have occurred in the drying stage. In addition, they produce biofertilizers with a discretization of more than $0 \%, 10 \%, 20 \%$, and $30 \%$. In the present study, the amount of biofertilizer is discretized in $5 \%$ increments. In any case, it is clear that an adequate addition of biofertilizer increases root length and fine roots. It would be important to further demonstrate that the appropriate proportion of vermicompost allows for the development of thicker roots as Esteves et al. (2020) claim. This is planned to be developed with another type of plant in a future investigation. The use of artificial intelligence in agriculture changes the way it is currently practiced (Dharmaraj and Vijayanand, 2018).

Finally, in Figure 12, a table with photographs of plants before soil removal can be observed. This analysis was not included in the results since, initially, it was not intended to measure visible fine roots for they were attached to the walls of the seedbed. Nevertheless, it is important to observe that the results visually agree with the projections estimated in Figure 10. The next research will be carried out with a larger sample and each plant will be photographed four times, every 90 degrees, in order to measure, with computer vision technology, the number of fine roots visible once they are removed from their seedbeds.

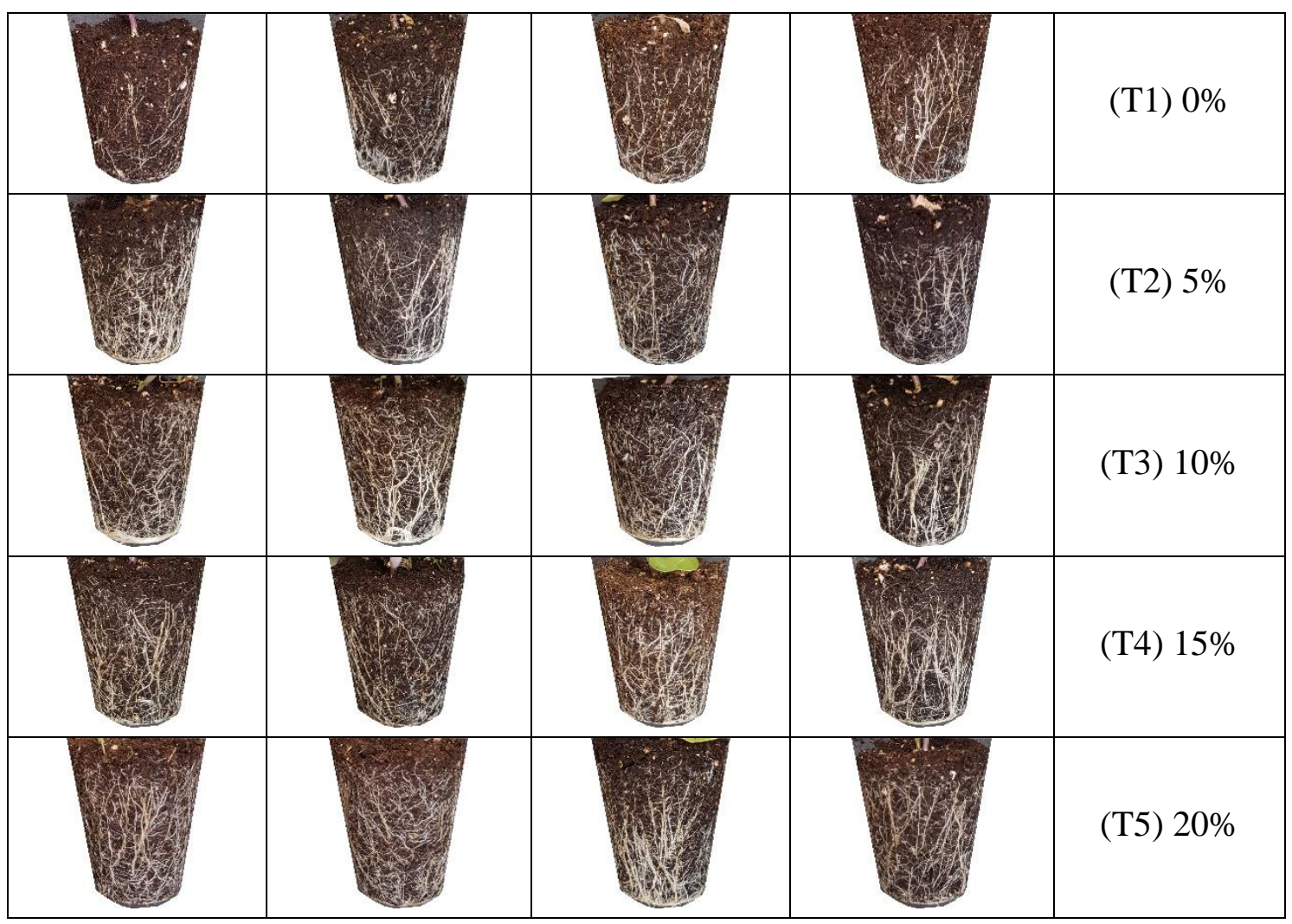




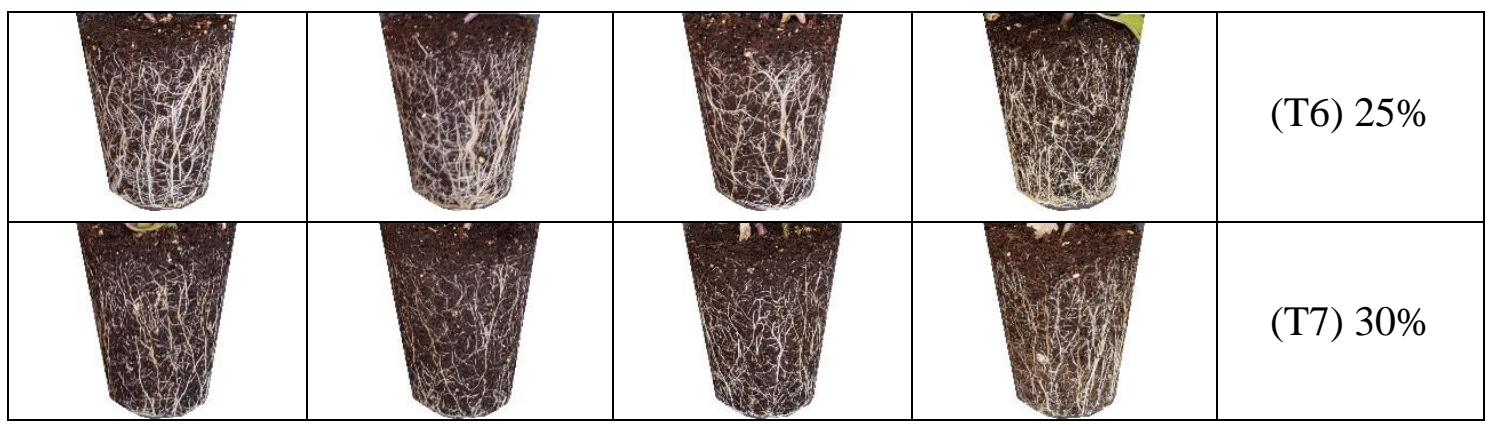

Figure 12. Root growth comparison between plant samples with different contents of biofertilizer before removing the soil

\section{Conclusions}

Microbial load fulfills essential functions in the rhizosphere. In order to achieve better root and root surface area development, it is important to add adequate amounts of biofertilizer; adding more than what is needed does not guarantee better development. The application of biofertilizers, enriched with beneficial microorganisms, benefits root development and plant growth. In addition, it improves soil characteristics, moisture retention, and nutrient availability. Vermicompost and compost together play an important role in water retention, nutrient supply, and soil characteristics. Mixing both materials with beneficial microorganisms (biofertilization) favors root length and root surface area. This research shows highly-accurate measurement results obtained by using computer vision technology. This also demonstrates that Python and OpenCV library are tools that can be used by the research community within the field of agricultural engineering to carry out high-performance experiments in less time. The use of non-destructive methods in real-time are the future research works, as detailed previously, in the discussion section on the visible roots attached to the walls of the seedbed. Additionally, this methodology is being tested in other crops in the study region, such as corn, wheat, among others.

Acknowledgements. This research has received funding from the Smart University 2.0 program, funded by "Tratamiento de residuos del faenamiento de aves, con aplicación de microorganismos benéficos."

\section{REFERENCES}

[1] Agarwal, P., Gupta, R., Gill, I. K. (2018): Importance of biofertilizers in agriculture biotechnology. - Sch. Res. Libr. 9: 1-3.

[2] Ahmadpour, R., Armand, N. (2020): Effect of ecophysiological characteristics of tomato (Lycopersicon esculentum L.) in response to organic fertilizers (compost and vermicompost). - Not. Bot. Horti Agrobot. Cluj-Napoca 48: 1248-1259.

[3] Altman, D. G. (1990): Practical Statistics for Medical Research. - Chapman \& Hall, London.

[4] Bannerjee, G., Sarkar, U., Das, S., Ghosh, I. (2018): Artificial intelligence in agriculture: a literature survey. - Int. J. Sci. Res. Comput. Sci. Appl. Manag. Stud. 7: 1-6.

[5] Betegón-Putze, I., González, A., Sevillano, X., Blasco-Escámez, D., Caño-Delgado, A. I. (2018): MyROOT: A novel method and software for the semi-automatic measurement of plant root length. - bioRxiv 1-23. 
[6] Bisht, J. K., Meena, V. S., Mishra, P. K., Pattanayak, A. (2016): Role of Biofertilizers in Conservation Agriculture. - In: Bisht J., Meena V., Mishra P., P. A. (eds.), Conservation Agriculture. Springer, Singapore, pp. 113-134.

[7] Böhm, W. (1979): Methods of Studying Root Systems. - Springer, Berlin.

[8] Chatterjee, R., Debnath, A., M. S. (2020): Soil Health. Soil Biology. - In: Giri, B., Varma, A. (eds.) Soil Health. Springer, Cham, pp. 69-88.

[9] Dharmaraj, V., Vijayanand, C. (2018): Artificial intelligence (AI) in Agriculture. - Int. J. Curr. Microbiol. Appl. Sci. 7: 2122-2128.

[10] Esteves, G. de F., de Souza, K. R. D., Bressanin, L. A., Andrade, P. C. C., Veroneze Júnior, V., dos Reis, P. E., da Silva, A. B., Mantovani, J. R., Magalhães, P. C., Pasqual, M., de Souza, T. C. (2020): Vermicompost improves maize, millet and sorghum growth in iron mine tailings. - J. Environ. Manage. 264: 1-11.

[11] Gopalakrishnan, S., Srinivas, V., Kumar, A. A., Umakanth, A. V., Addepally, U., Rao, P. S. (2019): Composting of sweet sorghum bagasse and its impact on plant growth promotion. - Sugar Tech 22: 143-156.

[12] Goswami, L., Nath, A., Sutradhar, S., Bhattacharya, S. S., Kalamdhad, A., Vellingiri, K., Kim, K.-H. (2017): Application of drum compost and vermicompost to improve soil health, growth, and yield parameters for tomato and cabbage plants. - J. Environ. Manage. 200: 243-252.

[13] Gouda, S., Kerry, R. G., Das, G., Paramithiotis, S., Shin, H.-S., Patra, J. K. (2018): Revitalization of plant growth promoting rhizobacteria for sustainable development in agriculture. - Microbiol. Res. 206: 131-140.

[14] Gupta, C., Prakash, D., Gupta, S., Nazareno, M. A. (2019): Role of Vermicomposting in Agricultural Waste Management. - In: Shah, S., Venkatramanan, V., Prasad, R. (eds.) Sustainable Green Technologies for Environmental Management. Springer, Singapore, pp. 283-295.

[15] Judd, L. A., Jackson, B. E., Fonteno, W. C. (2015): Advancements in root growth measurement technologies and observation capabilities for container-grown plants. Plants 4: 369-392.

[16] Kumar, S. M., Reddy, C. G., Phogat, M., Korav, S. (2018): Role of biofertilizers towards sustainable agricultural development: a review. - J. Pharmacogn. Phytochem. 7: 19151921.

[17] Lim, S. L., Wu, T. Y., Lim, P. N., Shak, K. P. Y. (2014): The use of vermicompost in organic farming: overview, effects on soil and economics. - J. Sci. Food Agric. 95: 11431156.

[18] Mahanty, T., Bhattacharjee, S., Goswami, M., Bhattacharyya, P., Das, B., Ghosh, A., Tribedi, P. (2017): Biofertilizers: a potential approach for sustainable agriculture development. - Environ. Sci. Pollut. Res. 24: 3315-3335.

[19] Nascente, A. S., De Filippi, M. C. C., Lanna, A. C., De Sousa, T. P., De Souza, A. C. A., Lobo, V. L. da S., Da Silva, G. B. (2017): Effects of beneficial microorganisms on lowland rice development. - Environ. Sci. Pollut. Res. 24: 25233-25242.

[20] Novoplansky, A. (2019): What plant roots know? Semin. - Cell Dev. Biol. 92: 126-133.

[21] Ottman, Timm (1984): Measurement of viable plant roots with the image analyzing computer. - Agron. J. 76.

[22] Paungfoo-Lonhienne, C., Redding, M., Pratt, C., Wang, W. (2019): Plant growth promoting rhizobacteria increase the efficiency of fertilisers while reducing nitrogen loss. - J. Environ. Manage. 233: 337-341.

[23] Schuurman, Goedewaagen (1965): Methods for the examination of root systems and roots. - Wageningen Cent. Agric. Publ. Doc. 37-41.

[24] Tian, H., Wang, T., Liu, Y., Qiao, X., Li, Y. (2020): Computer vision technology in agricultural automation-a review. - Inf. Process. Agric. 7: 1-19. 
[25] Tracy, S. R., Nagel, K. A., Postma, J. A., Fassbender, H., Wasson, A., Watt, M. (2020): Crop improvement from phenotyping roots: highlights reveal expanding opportunities. Trends Plant Sci. 25: 105-118.

[26] Tukey, J. W. (1977): Exploratory Data Analysis. - Pearson, London, pp. 131-160.

[27] Van Dusschoten, D., Metzner, R., Kochs, J., Postma, J. A., Pflugfelder, D., Bühler, J., Schurr, U., Jahnke, S. (2016): Quantitative 3D analysis of plant roots growing in soil using magnetic resonance imaging. - Plant Physiol. 170: 1176-1188.

[28] Villordon, A., LaBonte, D., Solis, J., Firon, N. (2012): Characterization of lateral root development at the onset of storage root initiation in 'Beauregard' sweetpotato adventitious roots. - HortScience 47: 961-968.

[29] Vives-Peris, V., De Ollas, C., Gómez-Cadenas, A., Pérez-Clemente, R. M. (2019): Root exudates: from plant to rhizosphere and beyond. - Plant Cell Rep. 39: 3-17.

[30] Wang, T., Rostamza, M., Song, Z., Wang, L., McNickle, G., Iyer-Pascuzzi, A. S., Qiu, Z., Jin, J. (2019): SegRoot: a high throughput segmentation method for root image analysis. - Comput. Electron. Agric. 162: 845-854.

[31] Weaver, J. E., Jean, F. C., Crist, J. W. (1922): Development and Activities of Roots of Crop Plants; a Study in Crop Ecology. - Carnegie Inst., Washington.

[32] Zha, J. (2020): Artificial intelligence in agriculture. - J. Phys. Conf. Ser. 1693: 1-6.

[33] Zuo, Y., Zhang, J., Zhao, R., Dai, H., Zhang, Z. (2018): Application of vermicompost improves strawberry growth and quality through increased photosynthesis rate, free radical scavenging and soil enzymatic activity. - Sci. Hortic. (Amsterdam) 233: 132-140. 